\section{Analysis of CD 133 Expression in Oral Leukoplakia}

Sir,

The term leukoplakia should be used to indicate a predominantly white lesion of the oral mucosa that cannot be wiped, other well-defined predominantly white lesions have been excluded clinically, histopathologically or by other diagnostic aids. ${ }^{1}$ Malignant transformation rate of oral leukoplakia varies from $17 \%$ to $35 \%$ as reported in different studies. Hence, a novel biomarker is required to identify this potentially malignant disorder. ${ }^{2}$

Studies have shown that the initiation, progression, including recurrence and metastasis of head and neck squamous cell carcinoma is related to the larger population of cancer stem cells. CD133 at first was described as a hematopoietic stem cells surface marker in the bone marrow; and now, its immune expressions have been used as a prognostic marker for survival of squamous cell carcinoma. ${ }^{2}$

This study was carried out at SEGi University, 15 cases of formalin fixed oral leukoplakia tissues with a clinical diagnosis of oral leukoplakia were obtained from the Malaysian oral cancer database and tissue bank system (MOCDTBS) at Oral Cancer Research and Coordinating Centre (OCRCC), University of Malaya, Malaysia and was approved by Medical Ethics Committee, Faculty of Dentistry, Reference number: DF OS1823/0077(L).

Cell membrane or cytoplasmic immune reactivity of epithelial cells were considered to indicate CD133 positive expression. Staining was classified as $<5 \%$ negative expression and $>5 \%$ positive expression. Intriguingly, no expression of CD133 was observed in any of these 15 cases of oral leukoplakia, suggesting that the assessed cases had minimal tumor initiation or had lower potential for malignant transformation.

The risk of transformation of leukoplakia to oral cancer remains difficult to assess, but some clinical factors are indicators of higher risk, like its duration, age, gender, site, size, habits like tobacco/alcohol, fungal infection. ${ }^{3}$ Dysplasia being the gold standard method to predict malignant potential, there is little agreement between pathologists regarding the epithelial dysplasia grading. Oral leukoplakia without epithelial dysplasia, having biochemical alterations but not exhibiting any morphological alterations, supports the actual clinical meaning of leukoplakia. These lesions may not be a simple hyperkeratosis, but can be a potentially dangerous lesion.4,5 Early detection of oral potentially malignant disorders can decrease the incidence of developing oral squamous cell carcinomas.

Further studies with larger samples may be required as there may be possible differences in expression of markers between individuals, race/region of the study, habits of tobacco/alcohol and the criteria used for diagnosis oral leukoplakia. 5

\section{REFERENCES}

1. van der Waal I. Historical perspective and nomenclature of potentially malignant or potentially premalignant oral epithelial lesions with emphasis on leukoplakia - some suggestions for modifications. Oral Surg Oral Med Oral Pathol Oral Radiol 2018; 125:577-81.

2. Liu $W$, Wu L, Shen XM, Shi LJ, Zhang CP, Xu LQ, et al. Expression patterns of cancer stem cell markers ALDH1 and CD133 correlate with a high risk of malignant transformation of oral leukoplakia. Int J Cancer 2013; 132:868-74.

3. Warnakulasuriya S, Johnson NW, Van Der Waal I. Nomenclature and classification of potentially malignant disorders of the oral mucosa. J Oral Pathol Med 2007; 36: 575-80.

4. Gomes CC, Gomez RS. Oral leukoplakia: What is achieved by surgical treatment? Ann J Oral Maxillofac Surg 2013; 1:1-3.

5. Brouns E, Baart JA, Karagozoglu KH, Aartman, Bloemena, van der Waal I. Malignant transformation of oral leukoplakia in a well-defined cohort of 144 patients. Oral Dis 2014; 20:e19-e24.

Anand Siddappa Tegginamani', Thomas George Kallarakkal ${ }^{2}$, HS Vanishreel and Khairiyah Binti Abd Muttalibl

1 Department of Dentistry, SEGi University Kota Damansara, Malaysia

2 Department of Dentistry, University of Malaya, Malaysia

Correspondence: Dr. Anand Siddappa Tegginamani, Department of Dentistry, SEGi University Kota Damansara, Malaysia

E-mail: anandsiddappa@segi.edu.my

Received: September 15, 2018; Revised: December 12, 2018; Accepted: February 04, 2019 